\title{
Getting to grips with a robotic arm
}

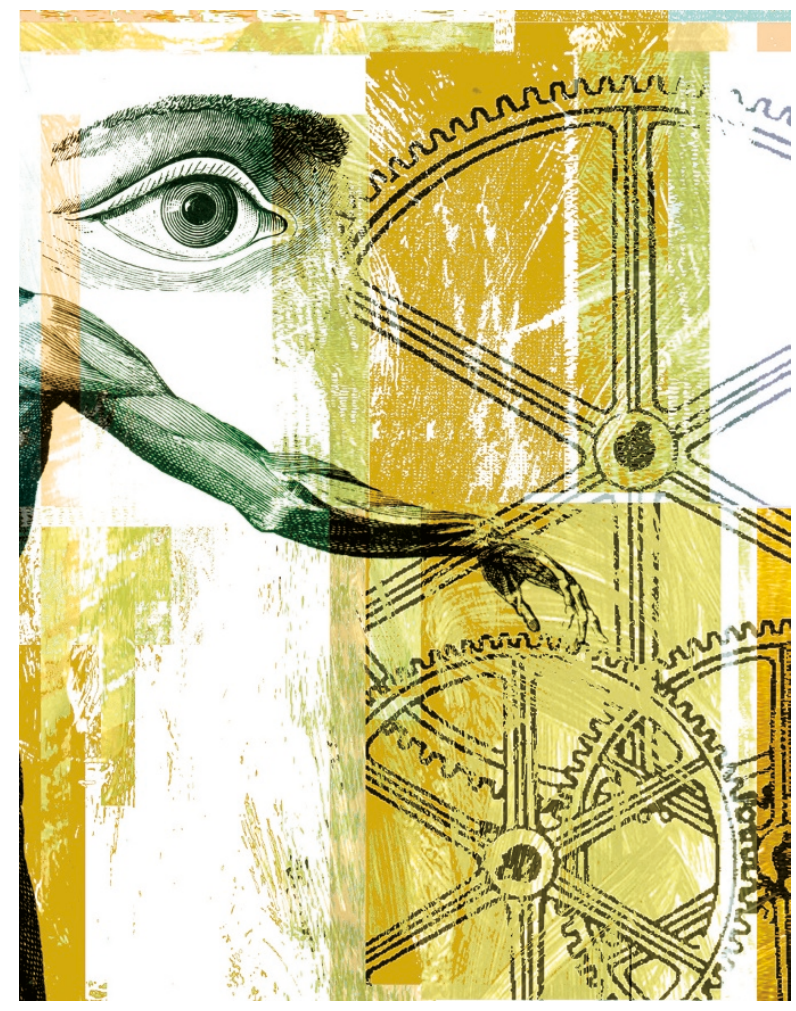

The possibility of using brain activity to control robotic devices gives hope to those whose mobility is severely reduced by neurological damage. Brain-machine interfaces have previously been used in animals and humans to move cursors on a screen or simple robotic devices using brain activity alone. Now, Schwartz and colleagues have taken the technology a step further, by training monkeys to operate a realistic prosthetic arm to feed themselves.

Arrays of microelectrodes were implanted into the primary motor cortex of two monkeys, allowing the authors to record the activity of approximately 100 neurons simultaneously. The authors first determined the tuning properties (the direction of arm movement that was associated with maximal firing of the neuron) of each recorded neuron as the monkeys watched a series of automated movements of a prosthetic jointed arm and 'hand' (a motorized gripper) while their own arms were restrained. These activity patterns were then fed into a simple algorithm that was later used to convert cortical firing patterns into movement of the prosthetic arm and hand.

The monkeys quickly learned to operate the arm with cortical activity alone and to complete a self-feeding task in which they repeatedly moved the arm towards a food target, grasped the food, moved the arm back to their mouth and then deposited the food. The arm movements appeared similar to natural arm movements and the monkeys were able to rapidly respond to changes in the position of the target, demonstrating the accuracy with which they could control the arm.

This study represents a significant advance in the technology of brain-machine interfaces: the task that was completed by the monkeys was more complicated and demanding than those that have previously been attempted and more closely resembled a real-life situation. Translation of the technology to human patients is eagerly awaited. However, some further improvements will be required, such as ways to prevent deterioration of the recorded signals over time and methods to allow sensory feedback from the prosthetic arm to the brain.

Katherine Whalley

ORIGINAL RESEARCH PAPER Velliste, M.

Perel, S., Spalding, M. C., Whitford, A. S. \& Schwartz, A. B. Cortical control of a prosthetic arm for self-feeding. Nature 28 May 2008 (doi:10.1038/nature06996) 